\title{
Motor excitability in bilateral moyamoya vasculopathy and the impact of revascularization
}

\author{
Gueliz Acker, MD,,2 Davide Giampiccolo, MD, ${ }^{1}$ Kerstin Rubarth, MSc, ${ }^{2,3}$ Robert Mertens, MD, ${ }^{1}$ \\ Anna Zdunczyk, MD, ${ }^{1}$ Juliane Hardt, Dipl-Psych, ${ }^{3-5}$ Daniel Jussen, MD, ${ }^{1}$ Heike Schneider, ${ }^{1}$ \\ Tizian Rosenstock, MD, ${ }^{1,2}$ Vera Mueller, MD, ${ }^{1}$ Thomas Picht, MD, ${ }^{1,6}$ and Peter Vajkoczy, MD'
}

\begin{abstract}
${ }^{1}$ Charité-Universitätsmedizin Berlin, corporate member of Freie Universität Berlin and Humboldt-Universität zu Berlin, Department of Neurosurgery, Berlin; ${ }^{2}$ Berlin Institute of Health at Charité-Universitätsmedizin Berlin, Berlin; ${ }^{3}$ CharitéUniversitätsmedizin Berlin, corporate member of Freie Universität Berlin and Humboldt-Universität zu Berlin, Institute of Biometry and Clinical Epidemiology, Berlin; ${ }^{4}$ University of Applied Sciences Hannover, Hochschule Hannover-University of Applied Sciences and Arts, Fakultät III, Department Information and Communication, Medical Information Management, Hannover; ${ }^{5}$ Department of Biometry, Epidemiology and Information Processing, WHO Collaborating Centre for Research and Training for Health in the Human-Animal-Environment Interface, University of Veterinary Medicine Hannover, Foundation, Hannover; and ${ }^{6}$ Cluster of Excellence: "Matters of Activity. Image Space Material," Humboldt University, Berlin, Germany
\end{abstract}

OBJECTIVE Motor cortical dysfunction has been shown to be reversible in patients with unilateral atherosclerotic disease after cerebral revascularization. Moyamoya vasculopathy (MMV) is a rare bilateral stenoocclusive cerebrovascular disease. The aim of this study was to analyze the corticospinal excitability and the role of bypass surgery in restoring cortical motor function in patients by using navigated transcranial magnetic stimulation (nTMS).

METHODS Patients with bilateral MMV who met the criteria for cerebral revascularization were prospectively included. Corticospinal excitability, cortical representation area, and intracortical inhibition and facilitation were assessed by nTMS for a small hand muscle (first dorsal interosseous) before and after revascularization. The clinically and/or hemodynamically more severely affected hemisphere was operated first as the leading hemisphere. Intra- and interhemispheric differences were analyzed before and after direct or combined revascularization.

RESULTS A total of 30 patients with bilateral MMV were examined by nTMS prior to and after revascularization surgery. The corticospinal excitability was higher in the leading hemisphere compared with the non-leading hemisphere prior to revascularization. This hyperexcitability was normalized after revascularization as demonstrated in the resting motor threshold ratio of the hemispheres (preoperative median 0.97 [IQR 0.89-1.08], postoperative median 1.02 [IQR $0.94-1.22$; relative effect $=0.61, p=0.03$ ). In paired-pulse paradigms, a tendency for a weaker inhibition of the leading hemisphere was observed compared with the non-leading hemisphere. Importantly, the paired paradigm also demonstrated approximation of excitability patterns between the two hemispheres after surgery.

CONCLUSIONS The study results suggested that, in the case of a bilateral chronic ischemia, a compensation mechanism between both hemispheres seemed to exist that normalized after revascularization surgery. A potential role of nTMS in predicting the efficacy of revascularization must be further assessed.

https://thejns.org/doi/abs/10.3171/2021.6.FOCUS21280

KEYWORDS moyamoya; navigated transcranial magnetic stimulation; nTMS; cortical excitability; revascularization

$\mathrm{M}$ OYAMOYA vasculopathy (MMV) is a rare cerebrovascular disease that is characterized by bilateral progressive stenoocclusion of basal cerebral arteries and represents one of the leading causes of stroke in children and young adults. ${ }^{1-3}$ Direct revascularization sur- gery via an anastomosis of the superficial temporal artery (STA)-to-middle cerebral artery (MCA; STA-MCA bypass) is indicated in MMV, as it has been shown to restore the impaired cerebrovascular hemodynamics and reduce recurrent ischemic and hemorrhagic stroke. ${ }^{4}$

ABBREVIATIONS ACVD = atherosclerotic cerebrovascular disease; CVRC = cerebrovascular reserve capacity; DSA = digital subtraction angiography; FDI = first dorsal interosseous; $\mathrm{LH}=$ leading hemisphere; $\mathrm{MCA}=$ middle cerebral artery; MEP = motor evoked potential; $\mathrm{MMD}=$ moyamoya disease; $\mathrm{MMV}=$ moyamoya vasculopathy; NH = non-leading hemisphere; $\mathrm{nTMS}$ = navigated TMS; RE = relative effect; RMT = resting motor threshold; SICI = short interval cortical inhibition; STA = superficial temporal artery; TIA = transient ischemic attack; TMS = transcranial magnetic stimulation.

SUBMITTED April 30, 2021. ACCEPTED June 23, 2021.

INCLUDE WHEN CITING DOI: 10.3171/2021.6.FOCUS21280. 
It is important to note that an ischemic brain injury leads to impaired excitability of the motor cortex. However, the brain has adaptive properties that allow a reorganization to compensate for the ischemic injury. ${ }^{5-7}$ By inducing electrical depolarization with a focal, rapid magnetic field induction, transcranial magnetic stimulation (TMS) allows brain function to be interrogated noninvasively. ${ }^{8,9}$ Moreover, its adoption into clinical practice has shown that it can be a valuable tool to predict recovery in stroke patients. ${ }^{10}$ However, targeting stimulation based on anatomical landmarks may limit spatial accuracy and thus bias evaluation of motor function. ${ }^{11}$ To overcome this issue, navigated TMS (nTMS) takes advantage of precise coupling between the patient's MRI study and head to offer individually targeted stimulation. ${ }^{11}$ While nTMS is considered a suitable tool for evaluating the neurophysiological changes after a stroke, ${ }^{7,12-14}$ there is a lack of studies analyzing the alterations in cortical excitability after blood flow restoration in ischemic brains. Our group previously investigated this with nTMS in 39 patients who underwent bypass surgery for atherosclerotic cerebrovascular disease (ACVD) ${ }^{12}$ In that study, cerebral revascularization led to improved motor excitability indicated by a lower resting motor threshold (RMT), intracortical disinhibition, and more focused motor cortical representation. ${ }^{12}$ This allowed us to introduce the concept of a "hibernating" brain in patients with unilateral chronic ischemia, based on the reversible impairment of motor cortical function after revascularization. However, MMV differs from ACVD in that it is bilateral, occurs more often in younger patients, and has unique patterns of cerebrovascular collateralization and cortical microvascularization. ${ }^{15,16}$ Nonetheless, the impairment of cerebrovascular reserve capacity (CVRC) and infarct patterns are comparable among both cerebrovascular diseases..$^{15}$

In this context, and to the best of our knowledge, cortical excitability has not been assessed before or after a revascularization procedure in patients with MMV. Therefore, the aim of our study was to analyze the cortical excitability and intracortical excitation-inhibition hemostasis in bilaterally diseased MMV patients and to describe changes after revascularization using nTMS.

\section{Methods \\ Study Design}

The study design was a prospective clinical case series. Adult patients diagnosed with MMV and scheduled for direct or combined revascularization at the Charité University Hospital, Berlin, between 2009 and 2014 were screened for study inclusion. Further inclusion and exclusion criteria are listed in the Supplementary Methods.

In the case of patients with bilateral MMV, we perform a staged treatment protocol. The side of the brain showing more severe clinical and/or hemodynamic impairment was treated first followed by revascularization of the second side, if needed, at 3 months or later. We defined the first treated hemisphere as the leading hemisphere (LH) and the other hemisphere as the non-leading hemisphere $(\mathrm{NH})$.

\section{Imaging Studies}

Radiological studies, including digital subtraction angiography (DSA), MRI (1.5 Tesla), and hemodynamic perfusion studies using acetazolamide-stimulated (Diamox) SPECT or $\mathrm{H}_{2} \mathrm{O}$-PET, were evaluated. Further details are described in Supplementary Methods. Briefly, ischemic lesions found in MRI were divided into two groups: group 1 included lacunar stroke and border zone infarction and group 2 included territorial infarctions, as described previously. ${ }^{17} \mathrm{CVRC}$ was considered maintained if the response to Diamox resulted in an increase in relative CVRC. If there was no increase after Diamox, or if a steal phenomenon occurred, then CVRC was considered impaired.

\section{Classifications}

Each hemisphere was classified separately according to the Suzuki staging system based on the present carotid artery pathology, extent of moyamoya vessels, and collateralization pathways, as described previously. ${ }^{18}$ Each hemisphere was also classified according to the Berlin grading system, ${ }^{19}$ as this enables a risk verification as described previously. ${ }^{20}$

\section{Navigated TMS}

All patients underwent biphasic single and paired-pulse nTMS for localization of motor eloquent cortex (Fig. 1). Further mapping details are described in the Supplementary Methods.

\section{Statistical Analysis}

Data analyses were performed with IBM SPSS version 25 (IBM Corp.), $\mathrm{R}$ version 4.0.3 (https://www.r-project. org), and SAS University Edition (SAS Institute Inc.). The statistical analyses and variables are explained in detail in Supplementary Methods.

\section{Results}

\section{Patient Characteristics}

A total of 32 MMV patients who had both a pre- and a postoperative nTMS examination were identified. Patients with unilateral moyamoya disease (MMD; $n=2)$ were excluded, resulting in a total of 30 patients who were included in the final analysis. More than half of the patients $(67 \%)$ were diagnosed with MMD, and 33\% of patients were diagnosed with moyamoya syndrome (Table 1). The majority of the patients were female $(n=21,70 \%)$. The mean age at the time of surgery was 40 years \pm SD 14 months. Clinical presentation was ischemic in 24 patients (80\%) and hemorrhagic in 4 patients (13.3\%). Other clinical presentations included seizure and headache (3\% each). Prior to surgery, only 5 patients experienced bilateral transient ischemic attacks (TIAs). All patients underwent a direct or combined revascularization procedure, including an STA-MCA bypass for the LH. Bypass patency was proven in all cases after the surgery by DSA or CTA. The median time interval between the revascularization surgery and the second nTMS was 3.5 months (range 4 days to 7.7 months). At the time of the second nTMS, none of the patients experienced any symptoms, and none were 


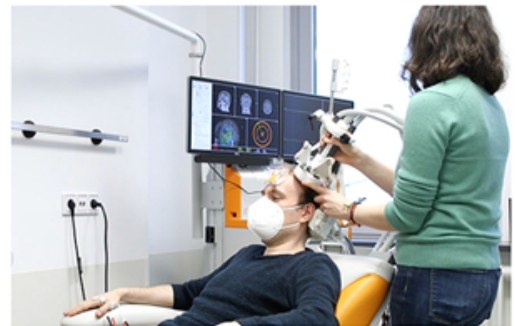

1) Corticospinal excitability

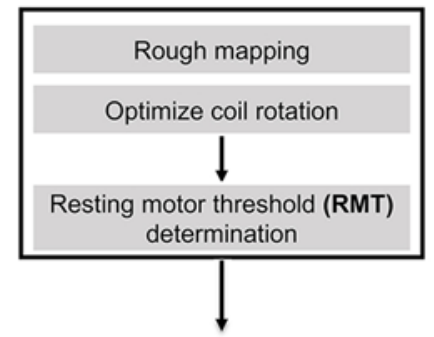

2) Cortical representation for the FDI muscle

Mapping and calculation of the representation area

3) Intracortical modulation

Short interval cortical inhibition [SICl] using an interstimulus interval (ISI) of $3 \mathrm{~ms}$

Intracortical facilitation [ICF] using ISIs between 5-17 ms

FIG. 1. Flowchart summarizing the cortical excitability assessment protocol with nTMS. 1) The preparation includes a rough and rotational mapping over motor cortex to localize the hotspot for the FDI and to optimize coil orientation. 2) The RMT was then used to map the representation area for cortical excitability of the FDI muscle and quantified by Voronoi tessellation. 3) Intracortical modulation was assessed by the paired-pulse paradigm. A subthreshold conditioning stimulus $(80 \%$ RMT) was administered before a test stimulus (120\% RMT) at the level of the FDI hotspot with different interstimulus interval.

found to have a new infarction on MRI. The majority of the bypass vessels demonstrated an excellent bypass function $(\mathrm{n}=19)$ in the DSA, while 1 patient did not have any relevant flow through the narrow bypass vessel. In this case, only the indirect revascularization-induced collateralization was present.

\section{Patient Imaging Characteristics: Infarct Profile and CVRC}

The majority of patients had a lacunar and/or border zone infarction in the LH $(24 / 29,82.8 \%)$. A territorial infarction occurred in 2 patients. A total of 18 patients $(18 / 29,62.1 \%)$ also had a lacunar and/or border zone infarction in the NH (Table 1).

Cerebral blood flow and CVRC were examined preoperatively in 28 patients. Of these, CVRC was impaired in $85.7 \%$ and in $35.7 \%$ of the LHs and NHs, respectively (Table 1). It is important that all 4 patients with sustained CVRC in the LH still had TIAs.

\section{Grading of Patients With MMV}

MMD was classified according to the Suzuki and the Berlin grading systems. The median Suzuki stages for the LHs and NHs were 4 and 2.5, respectively (Table 1). According to the Berlin grading, the majority of the LHs (19, $79.2 \%)$ were grade III, while only 7 NHs $(29.2 \%)$ were classified as grade III. The median Berlin grade was III and II for the $\mathrm{LH}$ and $\mathrm{NH}$, respectively.

\section{Navigated TMS Assessment}

RMT and the cortical representation area of the first dorsal interosseous (FDI) muscle were conducted to evaluate intra- and interhemispheric excitability. Preoperatively, the RMT values in the LH were lower than in the NH (median: LH 37\% [IQR 33\%-43\%]; NH 39\% [IQR $33 \%-47 \%]$; relative effect $[R E]=0.55, p=0.31, n=29$ ). After revascularization, the inverse was observed, with RMT values higher in the LH than in the $\mathrm{NH}$ (median: $\mathrm{LH}$ $43 \%$ [IQR 35\%-47\%]; NH 37\% [35\%-46\%]; RE $=0.44$, $\mathrm{p}=0.35, \mathrm{n}=29)$ (Figs. 2 and $3 \mathrm{~A}$ ). Of note, while $100 \%$ of stimulation output was insufficient to elicit motor evoked potentials (MEPs) in the $1 \mathrm{LH}$ preoperatively, these could be achieved after surgery. The cortical representation area of the FDI muscle was larger preoperatively in the LH (351.49 $\mathrm{mm}^{2}$ [IQR 269.01-509.25 $\mathrm{mm}^{2}$ ]) than in the $\mathrm{NH}$ $\left(250.10 \mathrm{~mm}^{2}\right.$ [IQR 184.72-461.32 $\left.\left.\mathrm{mm}^{2}\right]\right)(\mathrm{RE}=0.40, \mathrm{p}=$ $0.16, n=19)$. This was also reversed after revascularization with the cortical representation of the FDI muscle being smaller in the LH (243.41 $\mathrm{mm}^{2}$ [IQR 195.12-418.38 $\mathrm{mm}^{2}$ ]) than in the NH (353.5 $\mathrm{mm}^{2}$ [IQR 220.4-497.4 $\left.\left.\mathrm{mm}^{2}\right]\right)(\mathrm{RE}$ $=0.60, p=0.15, n=19)$ (Figs. $2 \mathrm{~B}$ and $3 \mathrm{C}$ ). When considering interhemispheric variations pre- and postoperatively, RMT in the LH increased after revascularization (preoperative 37\% [IQR 33\%-43\%] vs postoperative $43 \%$ [IQR 35\%-47\%]; RE $=0.63, \mathrm{p}=0.004, \mathrm{n}=29$ ), whereas RMT in the NH had no tendency to greater or lesser values pre- or postoperatively (39\% [IQR 33\%-47\%] vs $37 \%$ [IQR 35\%-46\%]; $\mathrm{RE}=0.50, \mathrm{p}=0.935, \mathrm{n}=29$ ). The cortical representation area of the LH tended to be larger preoperatively than postoperatively $\left(351.49 \mathrm{~mm}^{2}\right.$ [IQR 269.01-509.25 $\mathrm{mm}^{2}$ ] vs $245.08 \mathrm{~mm}^{2}$ [IQR 218.12-431.07 $\left.\left.\mathrm{mm}^{2}\right] ; \mathrm{RE}=0.42, \mathrm{p}=0.36, \mathrm{n}=19\right)$, whereas the area of the $\mathrm{NH}$ was preoperatively smaller $\left(247.63 \mathrm{~mm}^{2}\right.$ [IQR $183.51-$ $372.07 \mathrm{~mm}^{2}$ ] vs $356.1 \mathrm{~mm}^{2}$ [IQR 209.3-507.4 $\mathrm{mm}^{2}$ ]; RE = $0.63, \mathrm{p}=0.23, \mathrm{n}=17$ ). To account for potential interaction effects and confounding, we performed nonparametric unadjusted ANOVA as well as ANOVA adjusted for age and sex. The models for RMT indicated significant effects of the time point of the measurement ( $\mathrm{p}<0.01$, adjusted; $\mathrm{p}<$ 0.01 , unadjusted) and the interaction of the hemispheres with the time of measurement (pre- vs postoperative: $\mathrm{p}=$ 0.04 , adjusted; $p=0.02$, unadjusted). Similarly, the models for the cortical representation area also demonstrated a significant effect of the hemispheres with the time of measurement ( $\mathrm{p}=0.04$, adjusted; $\mathrm{p}=0.03$, unadjusted). Age and sex were not significant in both adjusted models. Furthermore, as both hemispheres were diseased, we 


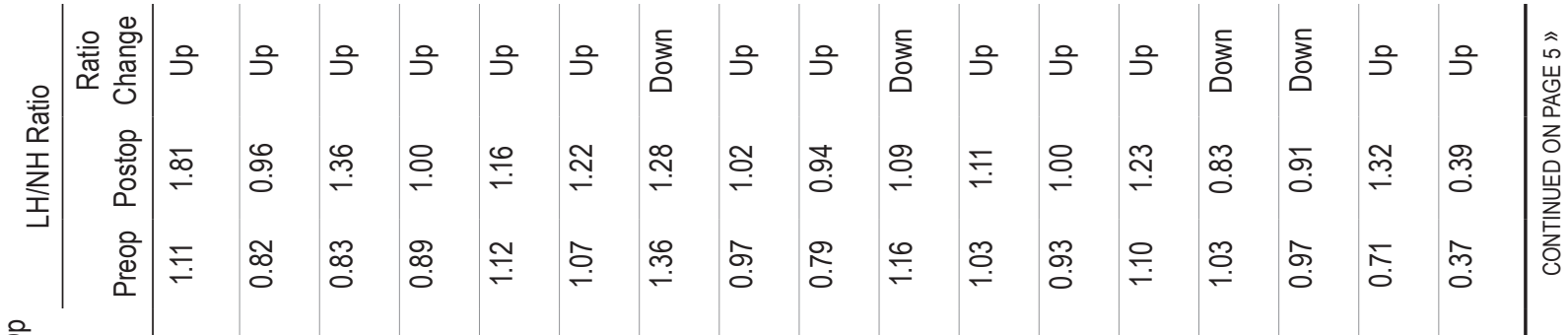

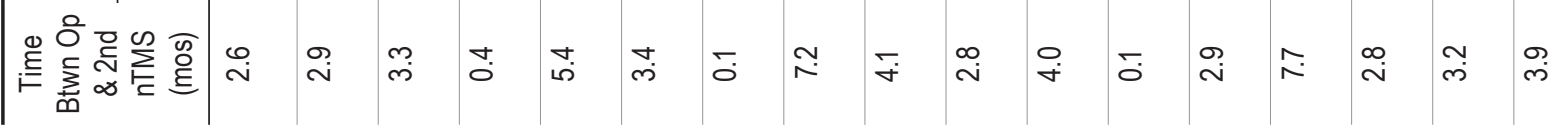

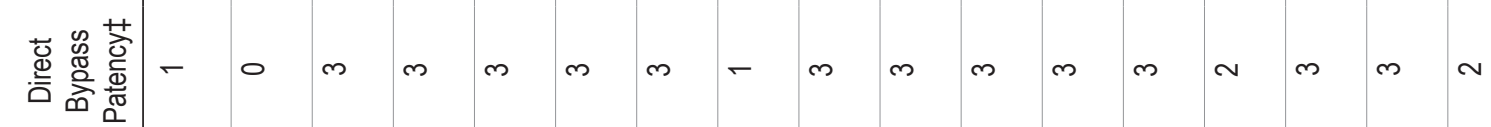

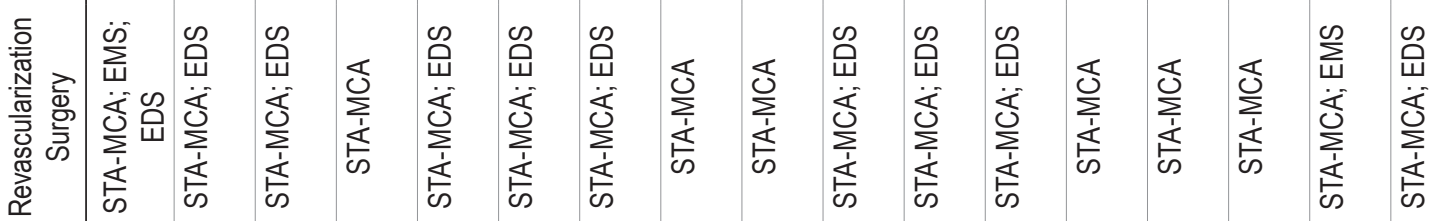

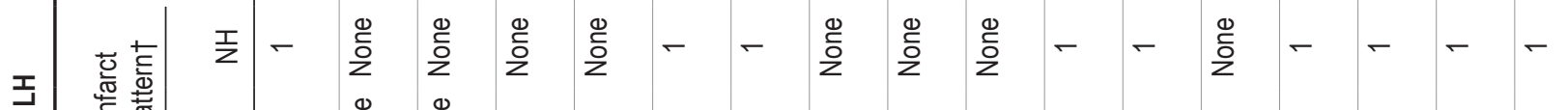
焉

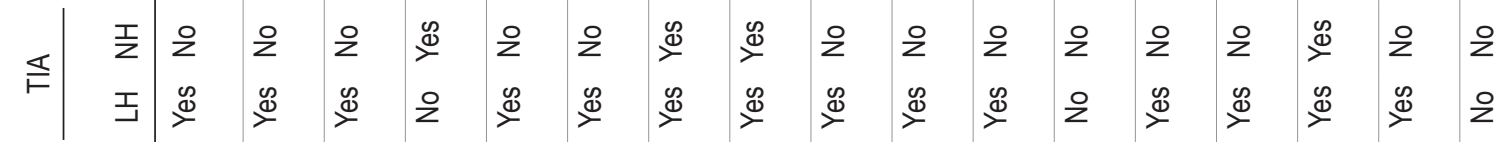

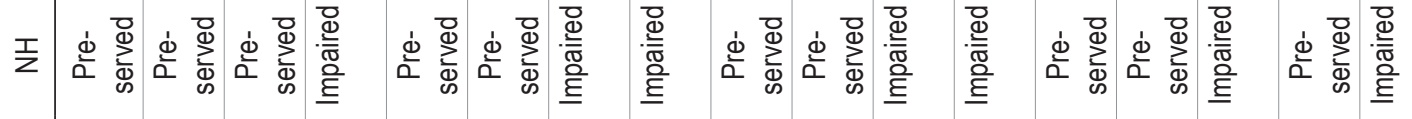

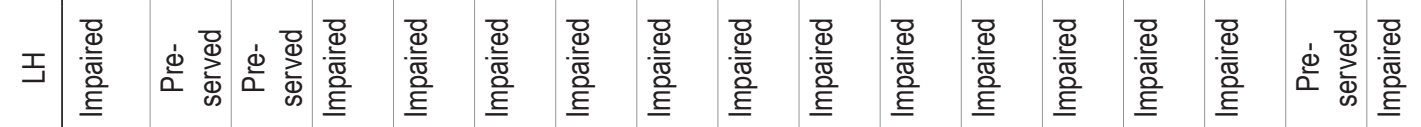

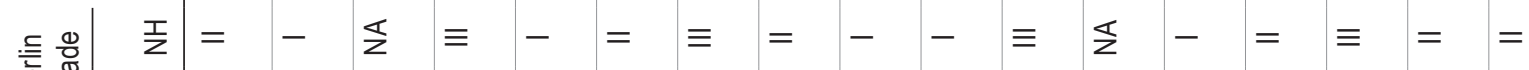

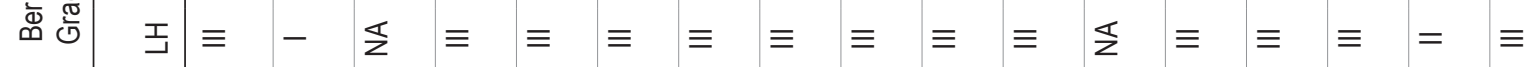

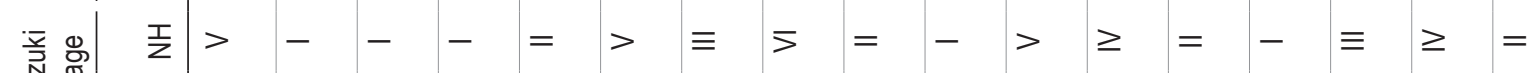

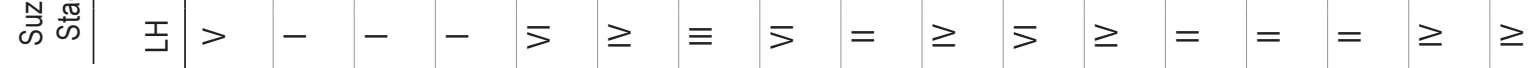

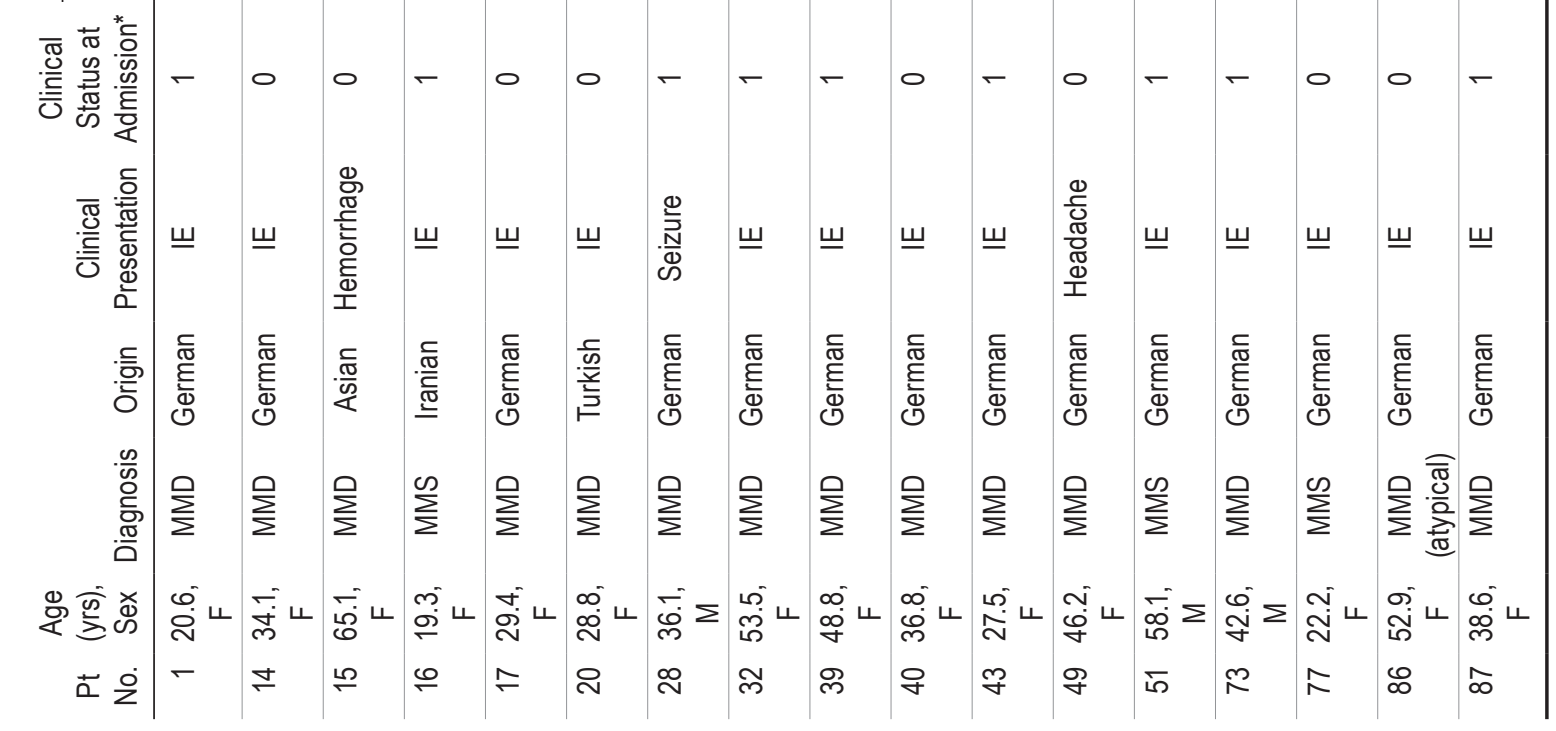




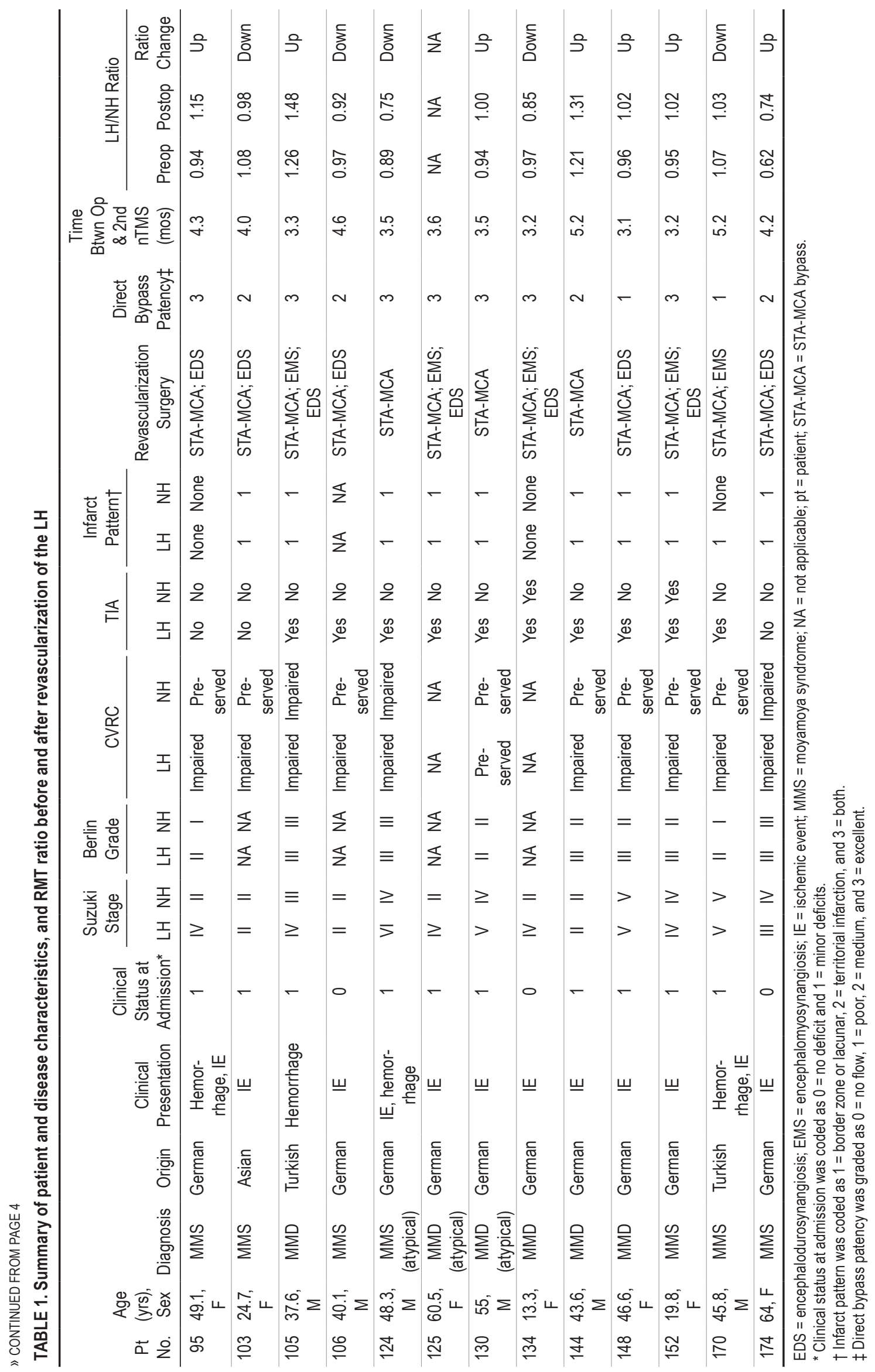



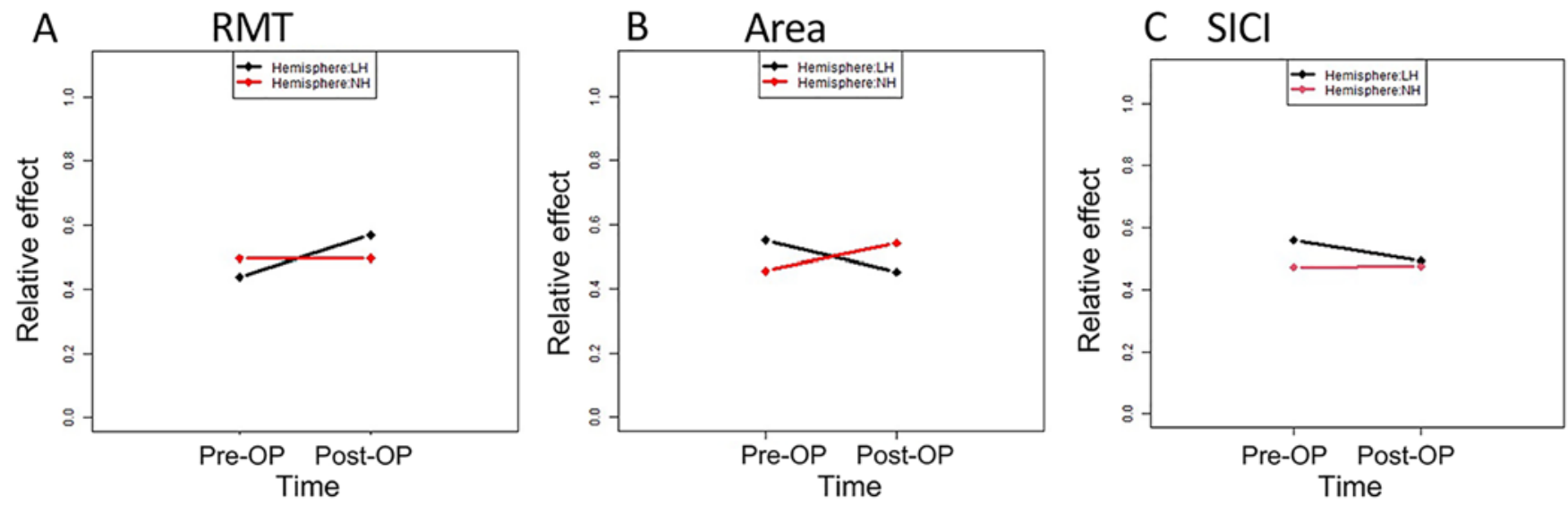

FIG. 2. A-C: RE estimates the probability that RMT (A), representation area $(B)$, and $\mathrm{SICl}$ measured at $3 \mathrm{msec}(\mathrm{C})$ from one hemisphere are smaller than those from the other hemisphere, pre- and postoperatively. A smaller RE indicates smaller values in the respective hemisphere at a certain time point. Consequently, we demonstrated here that the RMT and area in the LH reversed in comparison with $\mathrm{NH}$ after revascularization, while the $\mathrm{SICl}$ approximated after revascularization between the hemispheres.

analyzed the RMT ratio to judge the balance between the hemispheres. The RMT ratio $(\mathrm{LH} / \mathrm{NH})$ increased postoperatively to $1(0.97$ [IQR $0.89-1.08]$ vs 1.02 [IQR $0.94-$ 1.22]; $\mathrm{RE}=0.61, \mathrm{p}=0.03, \mathrm{n}=29$; Fig. 3B).

This outcome was due to the higher RMT in the LH prior to surgery and may indicate a normalization between the hemispheres after revascularization of the more affected hemisphere, as symmetric electrophysiological parameters have been demonstrated in healthy patients previously. ${ }^{21-23}$ Nevertheless, it must be highlighted that there were 9 patients in whom the ratio did not increase. However, we could not detect any differences between these and the rest of the patients with regard to several variables (Table 2). Supplementary Fig. 1 demonstrates the individual changes in RMT and the RMT ratio for each patient.
We sought to analyze motor excitability in depth using the paired-pulse paradigm to assess intracortical excitability before and after surgery. In paired-pulse stimulation, absolute values of baseline and differences between $3 \mathrm{msec}$ as cortical inhibition and $5 \mathrm{msec}, 7 \mathrm{msec}, 10 \mathrm{msec}$, and $17 \mathrm{msec}$ as cortical facilitation to baseline value $(0 / 120 \%)$ were examined.

Surgery did not affect baseline MEP amplitudes in either hemisphere (Fig. 4A and B; median pre- and postoperative: LH 521.2 $\mu \mathrm{V}$ [IQR 332.7-1258 $\mu \mathrm{V}$ ] and 452.4 $\mu \mathrm{V}$ [IQR 201.8-1377 $\mu \mathrm{V}$ ]; the relative effect was calculated with the complete cases: $\mathrm{RE}=0.51, \mathrm{p}=0.92, \mathrm{n}=22$; $\mathrm{NH} 745.5 \mu \mathrm{V}$ [IQR 287.2-1585 $\mu \mathrm{V}$ ] and 566.6 $\mu \mathrm{V}$ [IQR 323-1399 $\mu \mathrm{V}$ ]; the relative effect was calculated with the complete cases: $\mathrm{RE}=0.46, \mathrm{p}=0.47, \mathrm{n}=21$ ). Descriptive
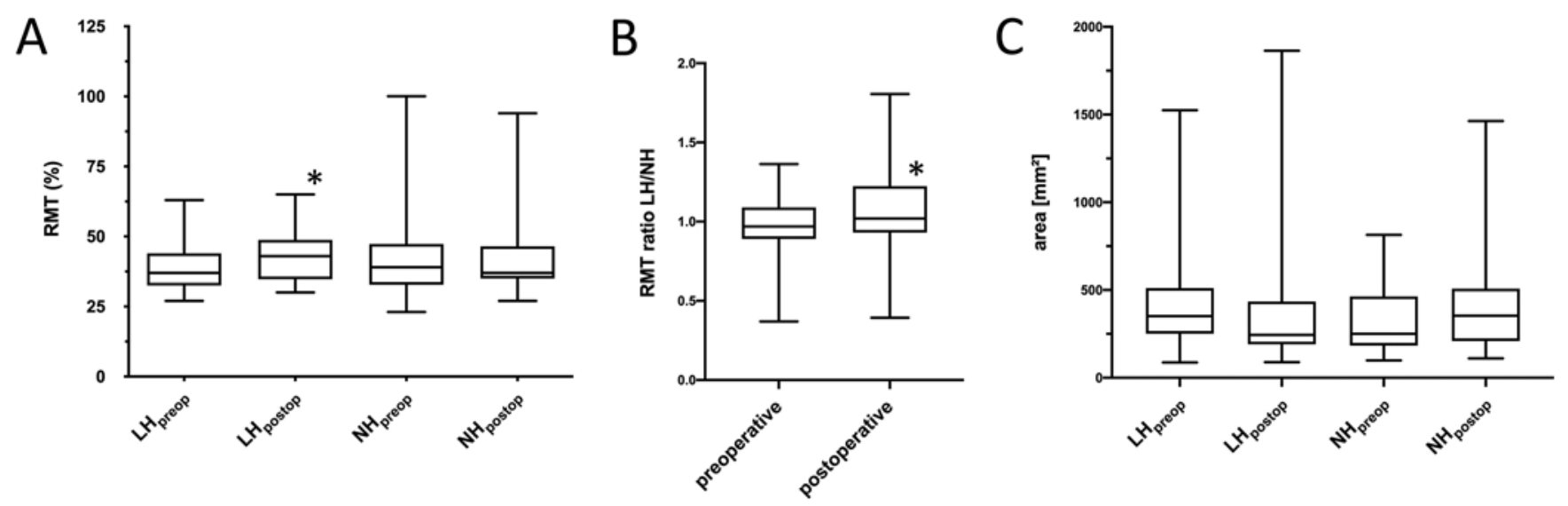

FIG. 3. Boxplots showing the RMT and the representation area A: RMT of both hemispheres before and after revascularization, demonstrating the increased RMT after revascularization in the LH (preoperative 37\% [IQR 33\%-43\%] vs postoperative $43 \%$ [IQR $35 \%-47 \%$ ]; ${ }^{2} \mathrm{RE}=0.63, p=0.004, n=29$ ). B: The RMT ratio increased statistically significantly after revascularization (preop 0.97 [IQR 0.89-1.08] vs postop 1.02 [IQR 0.94-1.22]; * $R E=0.61, p=0.03, n=29$ ). C: Representation area of the contralateral FDI muscle demonstrating comparable inter- and intrahemispheric values before and after surgery in the analysis with only minor differences. However, these minor differences were statistically significant in the nonparametric analysis, showing an effect of the hemispheres with the time of measurement $(p=0.04$, adjusted and $p=0.03$, unadjusted). LH preoperatively $n=19$, LH postoperatively $n=20$; and NH preoperatively $n=19$, NH postoperatively $n=19$. 
TABLE 2. Comparison of patient characteristics with an increase or decrease of the RMT ratio after revascularization

\begin{tabular}{|c|c|c|c|c|c|}
\hline \multirow[b]{2}{*}{ Variable } & \multicolumn{2}{|c|}{ Pts w/ Increased RMT Ratio } & \multicolumn{2}{|c|}{ Pts w/ Decreased RMT Ratio } & \multirow[b]{2}{*}{$\mathrm{p}$ Value } \\
\hline & No. of Pts & Value & No. of Pts & Value & \\
\hline Median age, yrs & 20 & $44.90(29.25-53.05)$ & 9 & $36.80(24.70-42.60)$ & 0.13 \\
\hline MMD diagnosis & 20 & $75 \%$ & 9 & $44 \%$ & 0.28 \\
\hline Female sex & 20 & $20 \%$ & 9 & $56 \%$ & 0.09 \\
\hline \multicolumn{6}{|l|}{ Median Suzuki stage } \\
\hline $\mathrm{LH}$ & 20 & $4(2-5)$ & 9 & $3(2-4)$ & 0.61 \\
\hline $\mathrm{NH}$ & 20 & $3.5(2-4.25)$ & 9 & $2(2-3)$ & 0.31 \\
\hline \multicolumn{6}{|l|}{ CVRC impaired } \\
\hline $\mathrm{LH}$ & 20 & $80 \%$ & 8 & $100 \%$ & 0.29 \\
\hline $\mathrm{NH}$ & 20 & $35 \%$ & 8 & $37.5 \%$ & $>0.99$ \\
\hline \multicolumn{6}{|l|}{ Median Berlin grade } \\
\hline $\mathrm{LH}$ & 18 & $3(3-3)$ & 6 & $3(3-3)$ & 0.73 \\
\hline $\mathrm{NH}$ & 18 & $2(1.25-2)$ & 6 & $2.5(1.25-3)$ & 0.63 \\
\hline \multicolumn{6}{|l|}{ Median RMT, \% } \\
\hline Preop & 20 & $37(33.5-43.5)$ & 9 & $37(33-42)$ & 0.80 \\
\hline Postop & 20 & $42(37.5-49.0)$ & 9 & $33(32-39)$ & 0.13 \\
\hline Median time btwn op \& nTMS, mos & 20 & $3.37(3.05-4.13)$ & 9 & $3.52(2.83-4.64)$ & 0.91 \\
\hline \multicolumn{6}{|l|}{ Presence of TIA } \\
\hline $\mathrm{LH}$ & 20 & $75 \%$ & 9 & $89 \%$ & 0.63 \\
\hline $\mathrm{NH}$ & 20 & $15 \%$ & 9 & $33 \%$ & 0.34 \\
\hline \multicolumn{6}{|l|}{ Presence of infarction } \\
\hline $\mathrm{LH}$ & 20 & $85 \%$ & 8 & $88 \%$ & $>0.99$ \\
\hline $\mathrm{NH}$ & 20 & $60 \%$ & 8 & $63 \%$ & $>0.99$ \\
\hline
\end{tabular}

analyses of intracortical excitability showed a trend toward motor disinhibition in the LH compared with the $\mathrm{NH}$ prior to surgery, which was reversed after surgery (Figs. 2C, 4A,
$4 \mathrm{~B}, 5 \mathrm{~A}$, and $5 \mathrm{~B})$. However, none of the alterations after revascularization reached a significant level based on the differences in the MEP amplitudes to baseline in a fur-
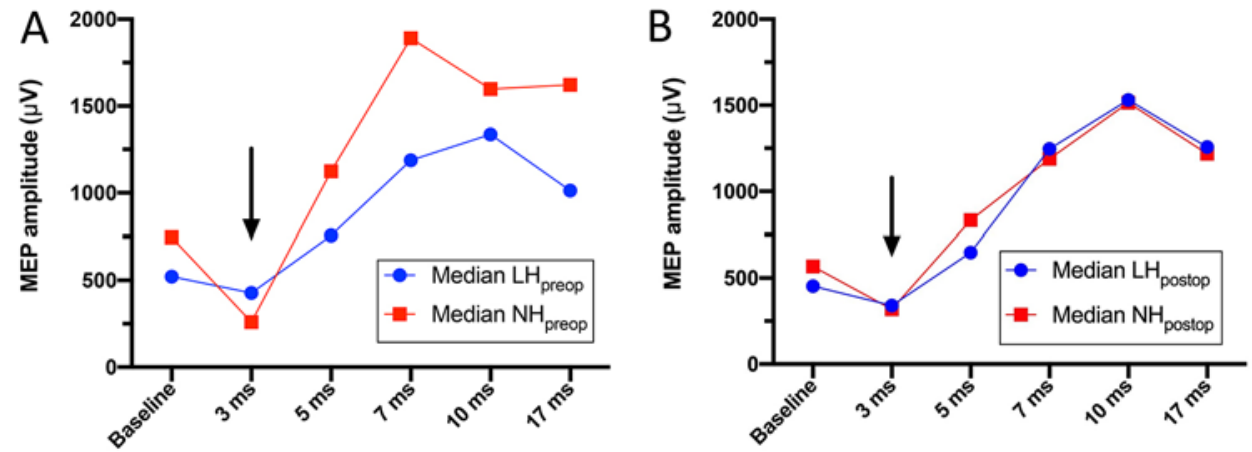

FIG. 4. The absolute MEP amplitudes at baseline and among different time intervals. A: Intracortical inhibition and facilitation assessed by paired-pulse stimulation, demonstrating slightly diminished intracortical inhibition by a 3-msec interval in comparison with the $\mathrm{NH}$ (arrow) that normalized after revascularization of the LH in the follow-up assessment after surgery. B: After revascularization, the two curves approach each other for all interval stimuli. LH preoperatively baseline: $n=24,3$ msec: $n=24,5$ msec: $n=18,7$ msec: $n=19,10$ msec: $n=20,17$ msec: $n=22 ;$ LH postoperatively baseline: $n=25,3$ msec: $n=24,5$ msec: $n=17,7$ msec: $n=17,10$ msec: $n=20,17$ msec: $n=22$. NH preoperatively baseline: $n=23,3$ msec: $n=23,5$ msec: $n=17,7$ msec: $n$ $=18,10$ msec: $n=19,17$ msec: $n=22 ;$ NH postoperatively baseline: $n=24,3$ msec: $n=23,5$ msec: $n=15,7$ msec: $n=15,10$ msec: $n=18,17$ msec: $n=20$. 

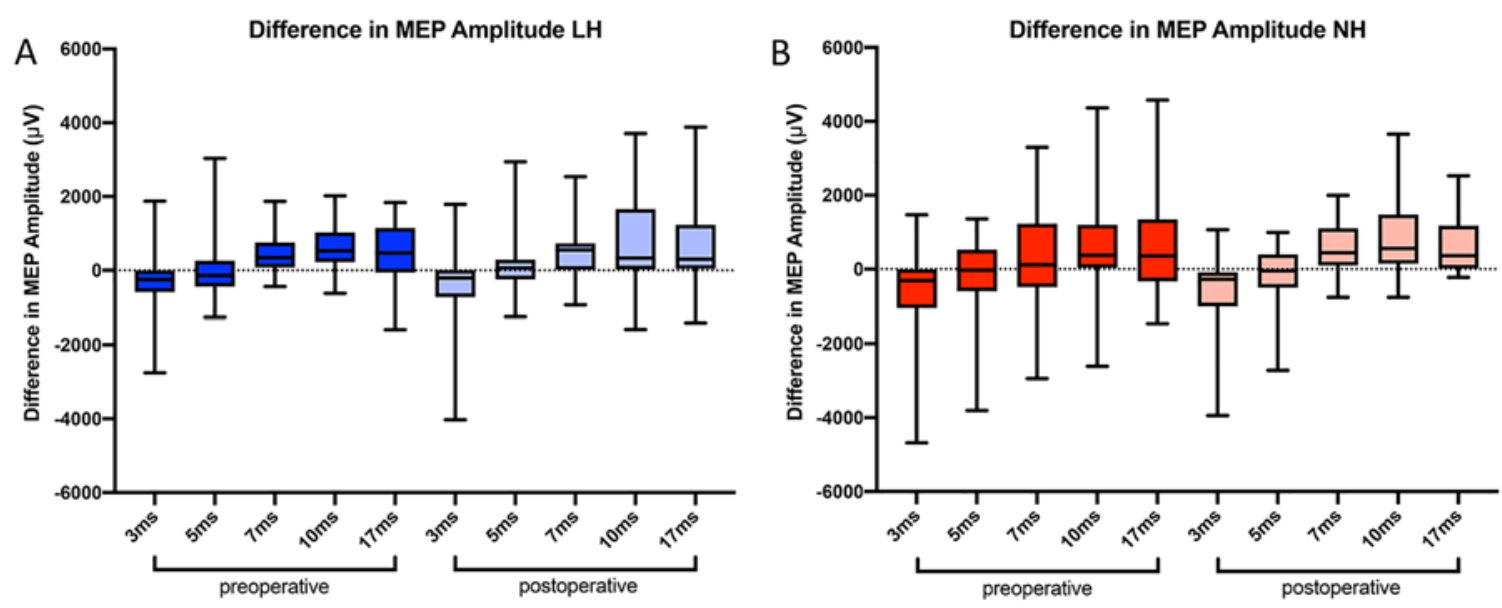

FIG. 5. Intracortical inhibition and facilitation assessed by paired-pulse stimulation. A and B: Boxplots showing the differences in MEP amplitudes for $\mathrm{LH}(\mathrm{A})$ and $\mathrm{NH}(\mathrm{B})$ in regard to the baseline among different interval stimuli. Due to higher variability, we did not observe any statistically significant changes. ( $\mathrm{SICl}$ was measured at $3 \mathrm{msec}$; while intracortical facilitation was measured at 5-17 msec.) Boxes represent IQRs, with the medians indicated by thick black lines. LH preoperatively 3 msec: $n=24,5 \mathrm{msec}: \mathrm{n}=$ 18, 7 msec: $n=19,10$ msec: $n=20,17$ msec: $n=22$; LH postoperatively 3 msec: $n=24,5$ msec: $n=17,7$ msec: $n=17,10$ msec: $n=20,17$ msec: $n=22$. NH preoperatively 3 msec: $n=23,5$ msec: $n=17,7$ msec: $n=18,10$ msec: $n=19,17$ msec: $n=22 ; N H$ postoperatively 3 msec: $n=23,5$ msec: $n=15,7$ msec: $n=15,10$ msec: $n=18,17$ msec: $n=20$.

ther analysis (Fig. 5A and B). In the adjusted analysis, the interstimuli interval (3-17 msec) was the only significant factor $(\mathrm{p}<0.001)$; this is expected physiologically, as explained in Supplementary Methods. Consequently, neither the hemisphere nor the revascularization effects were significant.

We further assessed a possible correlation of the RMT of each hemisphere with the presence of an infarction in MRI, presence of TIAs, disease grading systems, and the status of CVRC. In the LH, we observed a weak correlation between the presence of an infarction and both grading systems with RMT. Similarly, in the NH, we observed a weak correlation between TIAs and both grading systems with the RMT. Furthermore, a negative correlation was observed between the CVRC and the RMT of the NH (Supplementary Table 1). Additionally, we analyzed the correlation of both disease grading systems with the preoperative RMT ratio and found a medium correlation of the Berlin grade of the LH (Supplementary Table 1) with the preoperative RMT ratio. Finally, we saw that there was no correlation between the alteration of the RMT ratio after surgery (classified as up or down) and the time interval of the second nTMS examination.

\section{Discussion}

- Corticospinal Excitability in Stroke. The corticospinal excitability can be assessed using RMT, representation area, and short interval cortical inhibition (SICI). ${ }^{24} \mathrm{~A}$ reduced corticospinal excitability has frequently been described in unilateral chronic or acute stroke., $5,6,25$ The time frame of the analysis and the location of the stroke play a major role in the cortical reorganization. ${ }^{14,26}$ The interhemispheric compensation in unilateral stroke has not yet been clarified. ${ }^{24}$

- Corticospinal Excitability in ACVD. Decreased corti- cal excitability, represented by increased RMT in the affected hemisphere with recovery after revascularization, has been reported. The revascularization surgery led further to a disinhibition of the affected hemisphere. ${ }^{12}$

- Corticospinal Excitability in MMV. MMV is a unique bilateral chronic cerebrovascular disease in which the treatment of choice is revascularization. In MMV, there is a possible cerebrospinal reserve prior to surgery with normalization after it. As long as capacity is present, corticospinal excitability increases to compensate while RMT decreases and the cortical motor area enlarges. This can be normalized by a revascularization surgery. After revascularization, the RMT ratio between the two hemispheres approaches 1 , as a sign of balanced corticospinal excitability.

The present study describes the impact of direct or combined revascularization on motor cortical excitability in patients with bilateral MMV. Our results show that surgery in the most affected hemisphere significantly effects motor excitability in both hemispheres. Specifically, preoperative motor disinhibition in the LH, as suggested by decreased RMT and a larger representation area, was reversed by the revascularization with a normalized RMT ratio at follow-up (Fig. 6). Together with the well-established role in preventing both ischemic and hemorrhagic stroke in $\mathrm{MMV}^{4}$ these data confirm the role of bypass surgery in restoration of balanced cortical excitability.

\section{Navigated TMS in Stroke}

Navigated TMS has been increasingly applied to assess motor excitability in stroke patients; $;^{7,12-14}$ however, all these studies included only unilateral stroke patients. A reduced corticospinal excitability has frequently been described in patients with unilateral chronic or acute stroke 


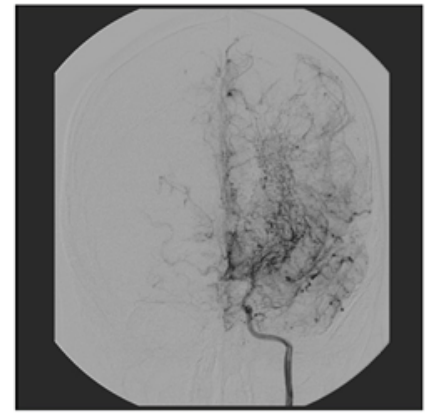

Hyperexcitability

- RMT $\downarrow$, Area $\uparrow$

- RMT Ratio $<1$

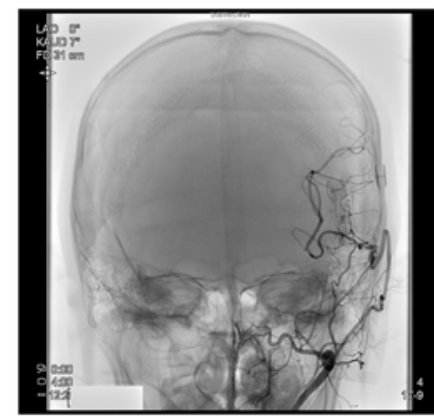

Normalization

$\underset{\text { Direct or combined Bypass }}{\longrightarrow}$
- RMT $\uparrow$, Area $\downarrow$

- RMT Ratio $\simeq 1$

FIG. 6. Coronal DSA images obtained in a patient with MMV before and after bypass surgery. Left: Prior to revascularization, a lower RMT with a larger motor representation area in the LH was observed. The RMT ratio between both hemispheres was $<1$, indicating an imbalance. Right: After revascularization, a higher RMT with a smaller representation area in the LH was identified. The RMT ratio between both hemispheres approaches 1 , which suggests normalization.

in comparison with the contralateral hemisphere. ${ }^{5,6,25}$ The corticospinal excitability has also been shown to have a prognostic value in stroke patients. ${ }^{10}$

Importantly, SICI may be altered bilaterally after unilateral stroke, suggesting an interhemispheric compensation. ${ }^{5,14}$ Ferreiro de Andrade and Conforto demonstrated that decreased short-interval intracortical inhibition correlated with better pinch strength and good motor recovery after stroke. ${ }^{27}$ In regard to disinhibition, Seo et al. stated that the unaffected hemisphere was more disinhibited than the affected hemisphere in the acute phase of mild to moderate stroke, while the affected hemisphere was more disinhibited in the subacute stage. ${ }^{28}$ The recent meta-analysis by McDonnell and Stinear confirmed an increased RMT in the affected hemisphere after stroke; however, hyperexcitability of the unaffected hemisphere or imbalanced interhemispheric inhibition remained debatable. ${ }^{24}$

\section{Effects of Revascularization in MMV Compared With ACVD}

MMV is a rare bilateral chronic cerebrovascular disease that differs from ACVD. ${ }^{15,16}$ While the indication to bypass surgery is generally disputed for treatment of chronic ACVD, it is rather unchallenged in cases of MMV to prevent further stroke. ${ }^{4}$ In this sense, revascularization has also been shown to improve functional outcome, confirming that treatment plays a role not only in stroke prevention but also in restoring function..$^{29}$ In the present study, we used nTMS to perform a specific quantitative assessment of chronic damage caused by bilateral MMV based on the corticospinal excitability.

Our group recently published results on motor cortical excitability prior to and after bypass surgery in a study using the same nTMS protocol in patients with unilateral ACVD. ${ }^{12}$ Strikingly, the results recapitulated preexisting literature in unilateral stroke with regard to reduced cortical excitability with an increased RMT. Here, the revascularization led to a recovery of the corticospinal excitability. Furthermore, the results showed that bypass surgery caused a motor disinhibition at $3 \mathrm{msec}$ instead of inhibi- tion. The result with regard to RMT is opposite to that in the current study, and, therefore, again underlines the differences in patients with bilateral MMV compared with those with unilateral chronic and acute stroke. Prior to the revascularization surgery, we detected a significantly reduced RMT in the more affected hemisphere (LH) that normalized after revascularization (Fig. 6). Supporting the greater motor excitability of the more severely affected hemisphere with lower RMT, we observed a larger FDI representation area preoperatively, as shown in the assessment of the REs. We observed only a tendency for an impairment in inhibition of the more affected hemisphere prior to revascularization. Overall, the revascularization of the more affected hemisphere led to a normalization of the excitability profiles of both hemispheres. On one hand, the RMT ratio between hemispheres normalized, and on the other hand, the paired-pulse paradigm presented an approximation of excitability patterns between the two hemispheres after surgery, as can be seen in their converging MEP curves. Although there are no predefined reference values for RMT, comparable excitable hemispheres and symmetric electrophysiological parameters for the hand representation were reported in healthy patients. ${ }^{21-23}$

Furthermore, we did not detect any significant correlations between RMT and TIAs, infarctions, MMV grading systems, or CVRC. Jussen et al. reported a correlation between CVRC and the difference in RMT between the affected and nonaffected hemispheres in ACVD. ${ }^{12} \mathrm{We}$ could not perform this analysis, as both hemispheres were affected in our study; instead, we repeated the correlation analysis with the preoperative RMT ratio without any significant correlations. In the current study, the majority of patients were investigated using SPECT, which can result in false-negative findings in detecting impairment in CVRC. In this regard, we recently demonstrated that ${ }^{15} \mathrm{O}-$ $\mathrm{H}_{2} \mathrm{O}$ PET enabled the detection of impaired CVRC in patients with MMV with negative ${ }^{99 \mathrm{~m} T c-H M P A O ~ S P E C T}{ }^{30}$ This might explain the missing correlation with RMT; on the other hand, this could also be a consequence of bilat- 
eral disease. Furthermore, a unique territory-specific perfusion pattern has been shown for MMV, including central preservation of cerebral blood flow that could support the compensation in the more affected hemisphere observed in this study. ${ }^{31}$

\section{A Possible Compensatory Cascade: Cerebrospinal Reserve}

Another chronic disease leading to progressive motor symptoms that can be addressed by neurosurgery is chronic degenerative myelopathy, in which a progressive stenosis impairs motor skills to the point of loss of function and can also lead to impaired corticospinal excitability. ${ }^{32}$ The analysis of motor excitability using nTMS in these patients suggests some kind of underlying cerebrospinal reserve. ${ }^{32}$ As long as a capacity for compensation is present, RMT decreases and cortical motor areas enlarge. In this scenario, easier recruitment of motor fibers from secondary motor areas enables preservation of motor function despite initial damage. Then, when compensatory reorganization becomes insufficient, damage also progressively involves secondary motor areas as the RMT increases and cortical motor areas shrink. We suppose that this model may also be applied to patients with MMV. With regard to the clinical differences, the MMV patient population is generally younger compared with the ACVD population.,12 In this study, the mean age was 40 years compared with the ACVD cohort with a mean age of 58 years, and the onset symptoms were not only ischemic. ${ }^{12}$ Moreover, the unique cerebrovascular collateralization and more intense cortical microvascularization of MMV seem to enable better brain function preservation. ${ }^{15,16}$ Consequently, patients with MMV might experience a more gradual impairment compared with overall stroke patient populations. In this regard, our patients with MMV possibly exemplify an earlier stage in the compensation cascade, while such mechanisms may already be exhausted by the time patients with ACVD are screened for revascularization. This is well reflected in the normalization of the RMT ratio after revascularization in patients with MMV, who may no longer need hyperexcitability in the more affected hemisphere to maintain function.

\section{Limitations}

The low number of patients is the main limitation of this study. Nevertheless, with regard to the rarity of MMV, 30 patients with follow-up account for a sufficient cohort to explore intra- and interhemispheric excitability changes before and after revascularization. Another limitation is the bias in patient selection, due to both the methodological burden of nTMS, which only allows for the examination of patients who are not severely paralyzed, and the lack of adolescent patients. The partially missing values due to the later extension of the nTMS protocol during the study period also represent a drawback, which was taken into account in the statistical analysis. Last but not least, the follow-up period after the revascularization was heterogeneous, ranging from 4 days to 7 months. We still decided to include the early postoperative phase since the direct revascularization improves the blood flow immediately, as illustrated in our review article. ${ }^{4}$ Consistent with this, we did not observe any correlation of RMT ratio alterations with the timing of the nTMS after the revascularization surgery. Since there was no control group without revascularization surgery, our focus was to assess the preoperative intra- and interhemispheric excitability hemostasis and to describe the effect of revascularization on it. Overall, this study was based on exploratory analyses to collect the first insights into this topic in patients with bilateral MMV. Therefore, the results need to be further confirmed in a larger cohort.

\section{Conclusions}

To our knowledge, this is the first study to assess the cortical excitability in a bilateral chronic cerebrovascular disease. Navigated TMS provided unique cortical excitability characteristics of patients with bilateral MMV before and after revascularization. Whether the preoperative RMT ratio could help select patients benefitting most from surgical treatment, or whether the normalization of the RMT ratio after revascularization could serve as a predictor of the long-term effectiveness of the bypass, has to be further investigated in a larger cohort.

\section{Acknowledgments}

Dr. Acker is a participant of the BIH-Charite Clinician Scientist Program that is funded by the Charité-Universitätsmedizin Berlin and the Berlin Institute of Health. Dr. Rosenstock is a participant of the digital junior clinician scientist program that is funded by the Charité-Universitätsmedizin Berlin and the Berlin Institute of Health. Both programs were initiated and led by Duska Dragun to enable resident physicians to pursue a career in academic research. With great sadness, we received the news that Dragun passed away on December 28, 2020. This publication is dedicated to her as a mentor, friend, role model, and stellar scientist. Noninvasive MRI measurements allowed a longitudinal study design and were supported by Charité 3RI Replace-Reduce-Refine. The authors acknowledge the support of the Cluster of Excellence: "Matters of Activity. Image Space Material," funded by the Deutsche Forschungsgemeinschaft (DFG; German Research Foundation) under Germany's Excellence Strategy-EXC 2025. We also thank Rob Hodson for proofreading the manuscript.

\section{References}

1. Research Committee on the Pathology and Treatment of Spontaneous Occlusion of the Circle of Willis; Health Labour Sciences Research Grant for Research on Measures for Infractable Diseases. Guidelines for diagnosis and treatment of moyamoya disease (spontaneous occlusion of the circle of Willis). Neurol Med Chir (Tokyo). 2012;52(5):245-266.

2. Acker G, Goerdes S, Schneider UC, Schmiedek P, Czabanka M, Vajkoczy P. Distinct clinical and radiographic characteristics of moyamoya disease amongst European Caucasians. Eur J Neurol. 2015;22(6):1012-1017.

3. Baba T, Houkin K, Kuroda S. Novel epidemiological features of moyamoya disease. J Neurol Neurosurg Psychiatr. 2008; 79(8):900-904.

4. Acker G, Fekonja L, Vajkoczy P. Surgical management of moyamoya disease. Stroke. 2018;49(2):476-482.

5. Berweck S, Walther M, Brodbeck V, Wagner N, Koerte I, Henschel V, et al. Abnormal motor cortex excitability in congenital stroke. Pediatr Res. 2008;63(1):84-88.

6. Hamzei F, Liepert J, Dettmers C, Weiller C, Rijntjes M. Two different reorganization patterns after rehabilitative therapy: an exploratory study with fMRI and TMS. Neuroimage. 2006;31(2):710-720. 
7. Liepert J, Greiner J, Dettmers C. Motor excitability changes during action observation in stroke patients. J Rehabil Med. 2014;46(5):400-405.

8. Ziemann U, Rothwell JC. I-waves in motor cortex. J Clin Neurophysiol. 2000;17(4):397-405.

9. Day BL, Dressler D, Maertens de Noordhout A, Marsden CD, Nakashima K, Rothwell JC, Thompson PD. Electric and magnetic stimulation of human motor cortex: surface EMG and single motor unit responses. J Physiol. 1989;412:449-473.

10. Smith MC, Stinear CM. Transcranial magnetic stimulation (TMS) in stroke: ready for clinical practice? J Clin Neurosci. 2016;31:10-14.

11. Ruohonen J, Karhu J. Navigated transcranial magnetic stimulation. Neurophysiol Clin. 2010;40(1):7-17.

12. Jussen D, Zdunczyk A, Schmidt S, Rösler J, Buchert R, Julkunen $\mathrm{P}$, et al. Motor plasticity after extra-intracranial bypass surgery in occlusive cerebrovascular disease. Neurology. 2016;87(1):27-35

13. Liepert J, Hamzei F, Weiller C. Motor cortex disinhibition of the unaffected hemisphere after acute stroke. Muscle Nerve. 2000;23(11):1761-1763.

14. Liepert J, Restemeyer C, Kucinski T, Zittel S, Weiller C. Motor strokes: the lesion location determines motor excitability changes. Stroke. 2005;36(12):2648-2653.

15. Czabanka M, Acker G, Jussen D, Finger T, Pena-Tapia P, Schubert GA, et al. Collateralization and ischemia in hemodynamic cerebrovascular insufficiency. Acta Neurochir (Wien). 2014;156(11):2051-2058.

16. Czabanka M, Peña-Tapia P, Schubert GA, Woitzik J, Horn P, Schmiedek P, Vajkoczy P. Clinical implications of cortical microvasculature in adult Moyamoya disease. J Cereb Blood Flow Metab. 2009;29(8):1383-1387.

17. Förster A, Szabo K, Hennerici MG. Pathophysiological concepts of stroke in hemodynamic risk zones-do hypoperfusion and embolism interact? Nat Clin Pract Neurol. 2008; 4(4):216-225.

18. Suzuki J, Takaku A. Cerebrovascular "moyamoya" disease. Disease showing abnormal net-like vessels in base of brain. Arch Neurol. 1969;20(3):288-299.

19. Czabanka M, Peña-Tapia P, Schubert GA, Heppner FL, Martus $\mathrm{P}$, Horn $\mathrm{P}$, et al. Proposal for a new grading of moyamoya disease in adult patients. Cerebrovasc Dis. 2011;32(1):41-50.

20. Kashiwazaki D, Akioka N, Kuwayama N, Houkin K, Czabanka M, Vajkoczy P, Kuroda S. Berlin grading system can stratify the onset and predict perioperative complications in adult moyamoya disease. Neurosurgery. 2017;81(6):986-991.

21. Bashir S, Perez JM, Horvath JC, Pena-Gomez C, Vernet M, Capia A, et al. Differential effects of motor cortical excitability and plasticity in young and old individuals: a transcranial magnetic stimulation (TMS) study. Front Aging Neurosci. 2014;6:111.

22. Zdunczyk A, Fleischmann R, Schulz J, Vajkoczy P, Picht T. The reliability of topographic measurements from navigated transcranial magnetic stimulation in healthy volunteers and tumor patients. Acta Neurochir (Wien). 2013;155(7):1309-1317.

23. Cicinelli P, Traversa R, Bassi A, Scivoletto G, Rossini PM. Interhemispheric differences of hand muscle representation in human motor cortex. Muscle Nerve. 1997;20(5):535-542.

24. McDonnell MN, Stinear CM. TMS measures of motor cortex function after stroke: a meta-analysis. Brain Stimul. 2017; 10(4):721-734.

25. Huynh W, Vucic S, Krishnan AV, Lin CS, Kiernan MC. Exploring the evolution of cortical excitability following acute stroke. Neurorehabil Neural Repair. 2016;30(3):244-257.

26. Manganotti P, Acler M, Zanette GP, Smania N, Fiaschi A. Motor cortical disinhibition during early and late recovery after stroke. Neurorehabil Neural Repair. 2008;22(4):396-403.
27. Ferreiro de Andrade KN, Conforto AB. Decreased shortinterval intracortical inhibition correlates with better pinch strength in patients with stroke and good motor recovery. Brain Stimul. 2018;11(4):772-774.

28. Seo HY, Kim GW, Won YH, Park SH, Seo JH, Ko MH. Changes in intracortical excitability of affected and unaffected hemispheres after stroke evaluated by paired-pulse transcranial magnetic stimulation. Ann Rehabil Med. 2018; 42(4):495-501.

29. Zhang M, Tang J, Liu N, Xue Y, Ren X, Fu J. Postoperative functional outcomes and prognostic factors in two types of adult moyamoya diseases. J Stroke Cerebrovasc Dis. 2020; 29(8):104846.

30. Acker G, Lange C, Schatka I, Pfeifer A, Czabanka MA, Vajkoczy P, Buchert R. Brain perfusion imaging under acetazolamide challenge for detection of impaired cerebrovascular reserve capacity: positive findings with ${ }^{15} \mathrm{O}$-water PET in patients with negative ${ }^{99 m}$ Tc-HMPAO SPECT findings. $J$ Nucl Med. 2018;59(2):294-298.

31. Schubert GA, Czabanka M, Seiz M, Horn P, Vajkoczy P, Thomé C. Perfusion characteristics of Moyamoya disease: an anatomically and clinically oriented analysis and comparison. Stroke. 2014;45(1):101-106.

32. Zdunczyk A, Schwarzer V, Mikhailov M, Bagley B, Rosenstock T, Picht T, Vajkoczy P. The corticospinal reserve capacity: reorganization of motor area and excitability as a novel pathophysiological concept in cervical myelopathy. Neurosurgery. 2018;83(4):810-818.

\section{Disclosures}

The authors report no conflict of interest concerning the materials or methods used in this study or the findings specified in this paper.

\section{Author Contributions}

Conception and design: Vajkoczy, Zdunczyk, Jussen. Acquisition of data: Acker, Giampiccolo, Zdunczyk, Jussen, Schneider, Rosenstock, Mueller. Analysis and interpretation of data: Acker, Giampiccolo. Drafting the article: Acker, Giampiccolo, Rubarth. Critically revising the article: Vajkoczy, Rosenstock, Picht. Reviewed submitted version of manuscript: Vajkoczy, Acker, Giampiccolo, Rubarth, Zdunczyk, Rosenstock, Picht, Hardt. Approved the final version of the manuscript on behalf of all authors: Vajkoczy. Statistical analysis: Rubarth, Hardt. Administrative/technical/material support: Mertens, Hardt, Schneider. Study supervision: Picht.

\section{Supplemental Information Videos}

Video Abstract. https://vimeo.com/586720144.

\section{Online-Only Content}

Supplemental material is available online.

Supplementary Methods, Tables, and Figure. https://thejns. org/doi/suppl/10.3171/2021.6.FOCUS21280.

\section{Correspondence}

Peter Vajkoczy: Charité-Universitätsmedizin Berlin, Germany. peter.vajkoczy@charite.de. 